\title{
GASTON FÉBUS OU LA CONSTRUCTION D’UNE LÉGENDE BÉARNAISE
}

\author{
Gaston Fébus ou a construção de uma lenda da \\ região de Béarn
}

\author{
Nadine Béague*
}

\begin{abstract}
The Béarn region of southern France, benefits from a rich history that is often personified by the history of Count Gaston Fébus (1331-1391). For several decades the region has been trying to develop local tourism. The 2015 restauration of the Morlanne castle owned by the Department of the Pyrénées-Atlantiques provided archeologists with the opportunity to research the castles of this strategically important region situated at the border between the kingdoms of France and England. This project also encouraged a discussion on the future of these sites and the role of the local population.
\end{abstract}

Keywords: tourism; castle; defensive architecture; medieval; France

\section{RÉSUMÉ}

Le Béarn est une région touristique du sud de la France qui bénéficie d'un riche passé historique incarné par Gaston Fébus (1331-1391). La réhabilitation du château de Morlanne, propriété du Département des Pyrénées-Atlantiques en 2015 nous donne l'occasion de mettre en perspective nos découvertes dans cette région riche en sites castraux située à la frontière des royaumes de France et d'Angleterre. La réflexion portera aussi sur le devenir de ces sites et l'implication des habitants.

Mots-clés: tourisme; château; architecture défensive; médiéval; France

* Archéologue Inrap. ITEM - EA 3002 IRSAM - Inrap Grand Sud Ouest. UPPA Avenue du Doyen Poplawski 64 000, Pau, France. Tél. : 06806021 92. nadine.beague@inrap.fr 


\section{RESUMO}

A região turística do Sul da França, o Béarn, é privilegiada por um rico passado histórico representado pela pessoa de Gaston Fébus (1331-1391). A reabilitação, em 2015, do castelo de Morlanne, propriedade do Département des PyrénéesAtlantiques, representa uma ótima oportunidade para os arqueólogos pesquisar esta região rica em sítios de castelos e localizada na fronteira entre os reinos da França e da Inglaterra. A presente reflexão também tratará do porvir destes sítios como da implicação dos residentes.

Palavras-chave: turismo; castelo; arquitetura defensiva; medieval; França

\section{Histoire et archéologie du Béarn, l' "invention » du concept de "château fébusien »}

Pour développer le tourisme, le Conseil Départemental des Pyrénées-Atlantiques prétend depuis les années 70 que «l'histoire de ce territoire ne commencerait vraiment qu'au Moyen Âge ", sous prétexte d'une absence quasi totale de sites archéologiques antiques. Cet argument employé par l'Office de Tourisme est erroné puisque des sites importants de l'Âge du Bronze à l'Antiquité tardive illustrant le potentiel archéologique du piémont pyrénéen ont été mis au jour par les archéologues bénévoles ou professionnels depuis plus de cinquante ans. Cette richesse patrimoniale est sous-exploitée, car il est plus confortable de s'appuyer sur l'architecture défensive médiévale et sur un personnage historique très médiatique : Gaston III de Foix, dit Fébus (1331-1391). Ce seigneur fut à la tête d'un comté (Foix) et de vicomtés (dont celle de Béarn) situées sur le passage des armées entre Atlantique et Méditerranée, entre Nord et Sud des Pyrénées pendant la guerre de Cent Ans. Ce prince qui se proclama lui-même "Fébus" (1358) dans ses actes, ses florins d'or et ses forteresses (Febus me Fe) monnaya sa neutralité face aux grandes monarchies, et créa le concept d'autonomie du Béarn. 


\section{Figure 1}
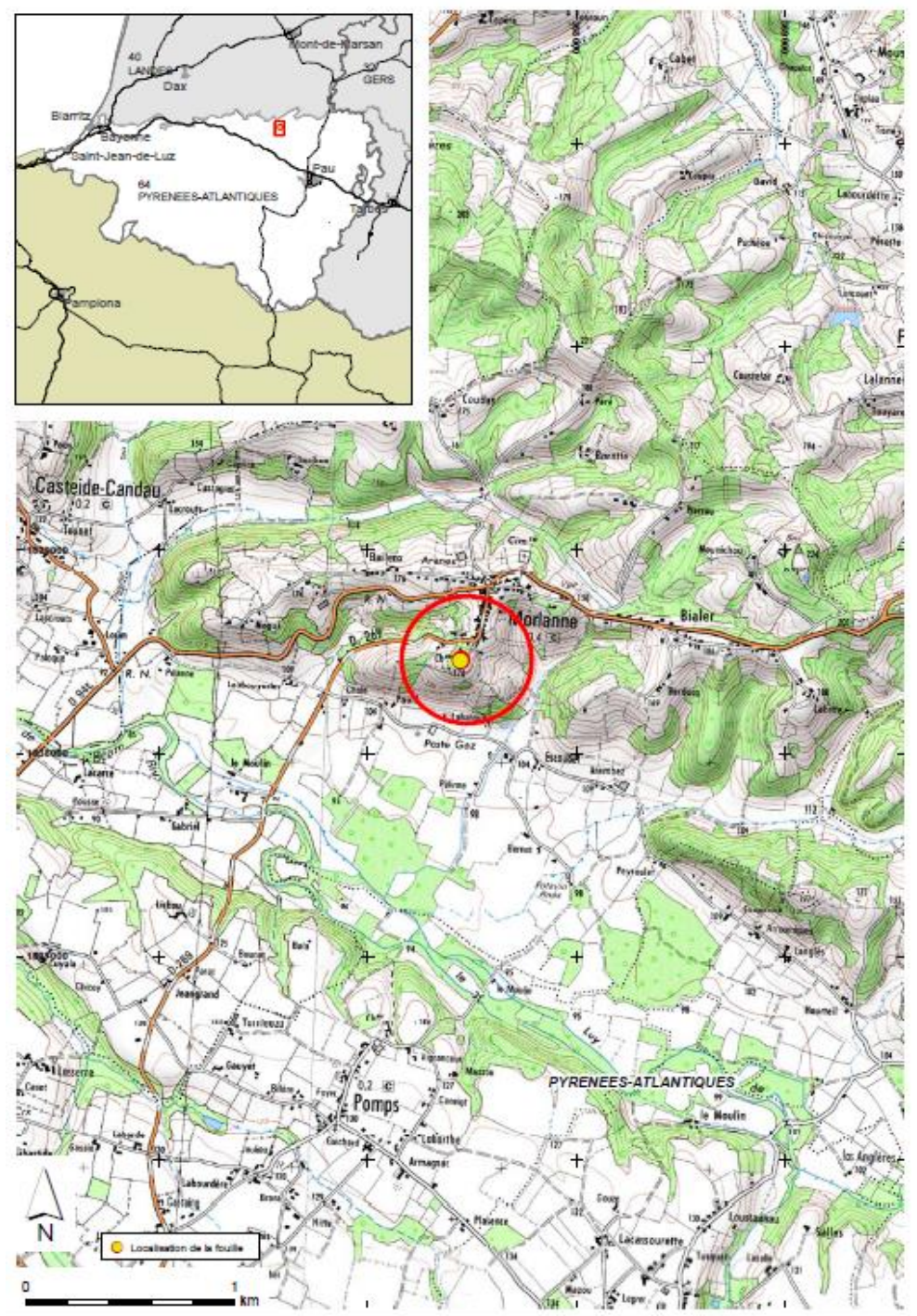

Localisation du site au 1/25000 @ Inrap 
Selon les historiens Raymond Ritter (1953) et Pierre TucooChala (1993) ses outils de dissuasion furent plus ses châteaux et fortifications qu'une armée bien équipée et efficace. Raymond Ritter (1894-1974) était avocat, mais nourrissait une véritable passion pour l'histoire et l'archéologie et il publia de nombreux ouvrages sur l'architecture militaire du Moyen Âge. Très sensible à la protection $\mathrm{du}$ patrimoine dès les années 30 , il s'efforça d'alerter l'opinion publique dans la presse face à la disparition de monuments des Pyrénées-Atlantiques ayant servi de carrière de pierres après la Révolution française de 1789. Après avoir acheté le château de Morlanne en ruines en 1969, Raymond Ritter lui redonne un caractère de forteresse médiévale sans bénéficier d'aucune aide financière et ayant à l'esprit un "ailleurs imaginé dans le lointain des siècles" (CHADEFAUD, 1988), il l'intègre dans son itinéraire touristique appelé "Route des Preux et des Maréchaux". En 1971, il reçoit le 2e prix de l'émission "Chefs d'œuvre en péril", puis lègue le château au Département des Pyrénées-Atlantiques en 1974.

À une époque où l'archéologie médiévale n'existait pas encore, Ritter et Tucoo-Chalaa, (1924-2015) professeur d'université spécialiste de l'histoire du Béarn, président de la Société des Sciences, Lettres et Arts de Pau ainsi que de l'Académie du Béarn, ont forgé l'histoire d'un Gaston Fébus construisant à partir des années 1370 une ligne de forteresses contre les Anglais. Selon eux, des châteaux relevant tous d'une même conception architecturale s'imposèrent aux endroits stratégiques du Béarn. Le château dit "fébusien" se composait d'une enceinte polygonale contre laquelle étaient adossés des logis et communs disposés en continu autour d'une cour intérieure, d'une tour quadrangulaire à cheval sur cette enceinte, et d'un fossé qu'enjambait un pont-levis donnant accès à l'entrée. Gaston Fébus aurait simplifié au maximum le plan architectural du château, économisé en achetant de grandes quantités de matériaux de qualité constante produits sur place (la brique), en ne faisant pas appel à des artisans spécialisés, mais en utilisant une main-d'œuvre locale.

Le château de Morlanne aurait été construit en briques vers 1373-1374, mais, comme il a subi d'importants travaux à différentes époques pour le rendre plus confortable, il est à présent difficile de se faire une idée de ce à quoi il pouvait ressembler à l'origine. En 2012, le Conseil Départemental des Pyrénées-Atlantiques, devenu 
propriétaire du château à la mort de Raymond Ritter, a décidé de le réhabiliter. La Direction Régionale des Affaires Culturelles de nouvelle-Aquitaine a délivré un permis de réhabilitation comportant la création d'un musée de $400 \mathrm{~m} 2$, la réalisation d'un accès sécurisé des visiteurs au donjon via le chemin de ronde en prenant en compte le public handicapé, tout en sous-entendant que le caractère " fébusien » du château devait être respecté. Il y est stipulé qu' «il parait indispensable de faire une analyse critique des constructions intra-muros avant de reconstruire un état néomoderniste s'ajoutant à l'état néo-médiéval ».

\section{Figure 2}

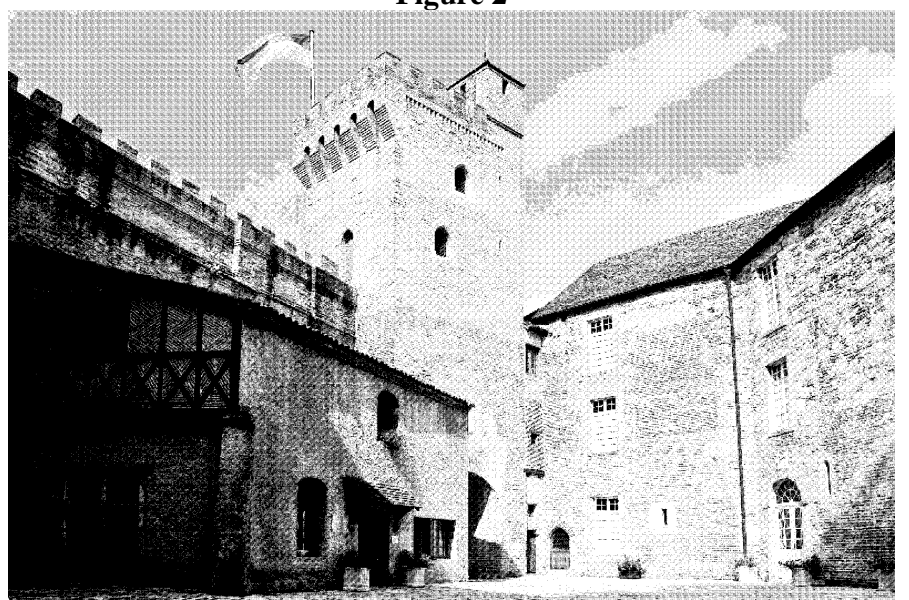

Cour intérieure du château avant réhabilitation@( Photo N. Béague, Inrap

L'opération archéologique menée au château de Morlanne entre 2012 et 2015 (BÉAGUE, 2016) a donc eu pour cadre la restauration et l'amélioration des espaces ouverts au public. La surveillance de travaux pour l'aménagement $\mathrm{du}$ réseau d'assainissement et le creusement de la cage d'ascenseur assortis d'un relevé partiel du bâti à l'occasion du décrépissage des élévations devaient permettre d'établir un bilan stratigraphique et archéologique du site. Le sous-sol du château n'étant pas documenté à ce jour d'un point de vue archéologique, c'était l'occasion unique de restituer 
l'image des bâtiments originels adossés à la face interne de la muraille tels qu'ils étaient avant les restructurations et restitutions des différentes époques, et ainsi, de faire le point sur les hypothèses historiques et architecturales émises à propos de cet édifice. Le diagnostic réalisé dans la cour intérieure du château a débouché sur une fouille préventive en raison d'un résultat très positif.

Figure 3

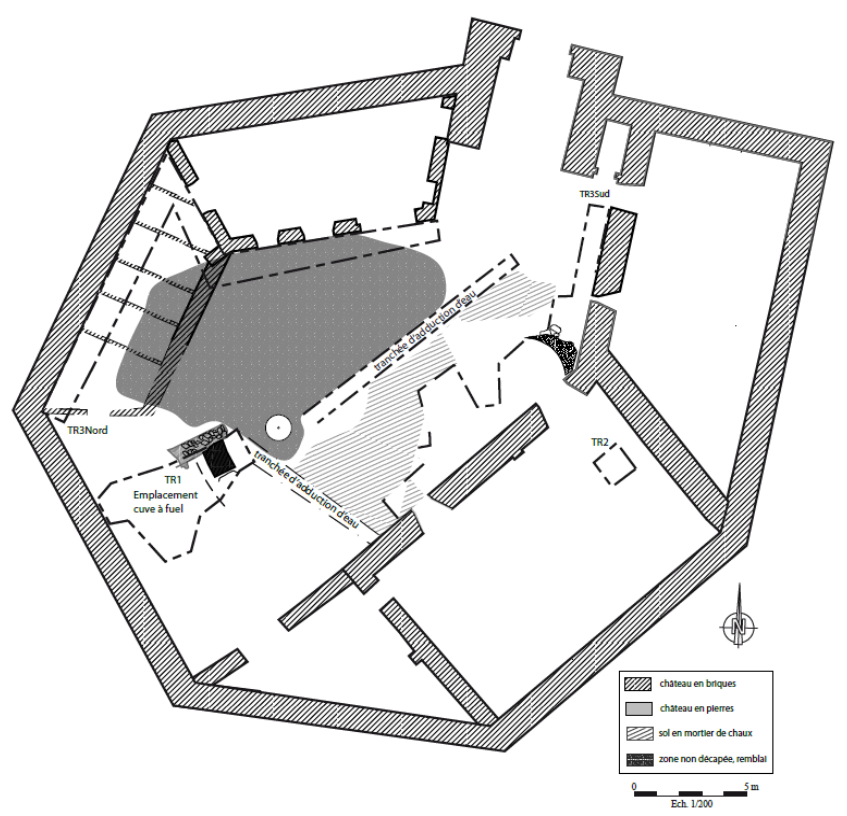

Plan de détail des tranchées de diagnostic (C) DAO F. Bernard et N. Béague, Inrap

\section{Les vestiges découverts}

La fouille archéologique préventive a permis de mettre au jour les vestiges d'un bâtiment construit en pierres de taille antérieur 
au château en briques. L'interprétation de ces vestiges est rendue difficile par une récupération des matériaux de construction par les bâtisseurs du château de l'époque de Gaston Fébus. Les éléments très arasés d'une base d'escalier sous le perron actuel du château et d'une cour intérieure circulaire permettent de proposer la restitution du premier château de Morlanne tel que nous le notons dans les prochains paragraphes.

\section{Figure 4}

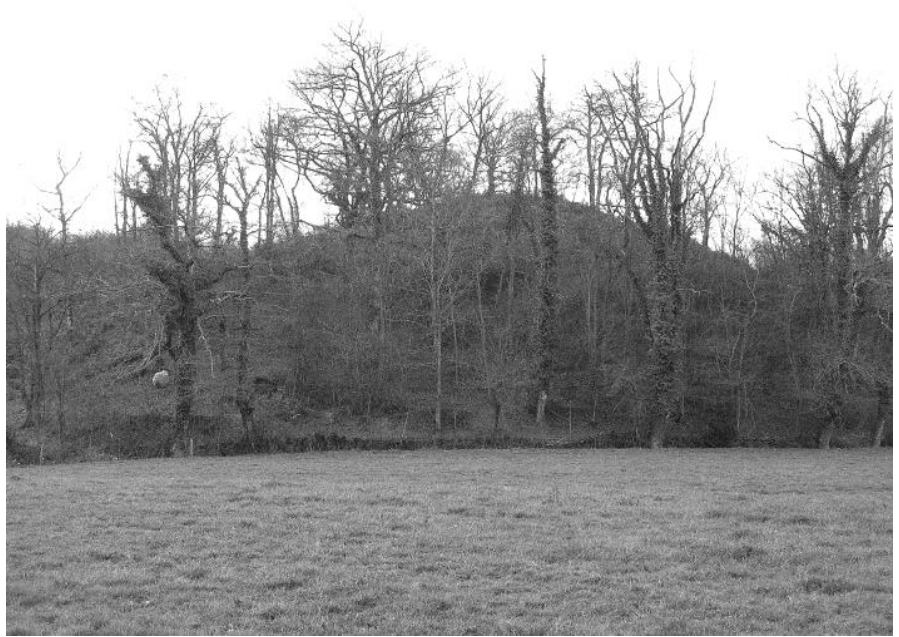

Motte féodale d'Haurou, commune de Castillon-d'Arthez (C) N. Béague, Inrap

Une imposante couche de remblai argileux observée dans le sondage réalisé à l'intérieur du château actuel pour le creusement de la cage d'ascenseur atteste qu'une motte constituée de main d'homme formait l'assise d'un château en terre et en bois ou "moated site" au XIe ou XIIe siècle (figure 4). Le parement externe d'une maçonnerie en pierres de grand appareil mis au jour témoigne qu'une fortification en pierres succède à ce premier état en terre et en bois, peut-être au XIIe siècle. Cette maçonnerie dégagée sur une longueur d'1,10 m et se prolongeant sous le château actuel pourrait se rapporter à une tour 
maîtresse polygonale ou à une salle fortifiée pour ce que l'on peut en juger.

Figure 5

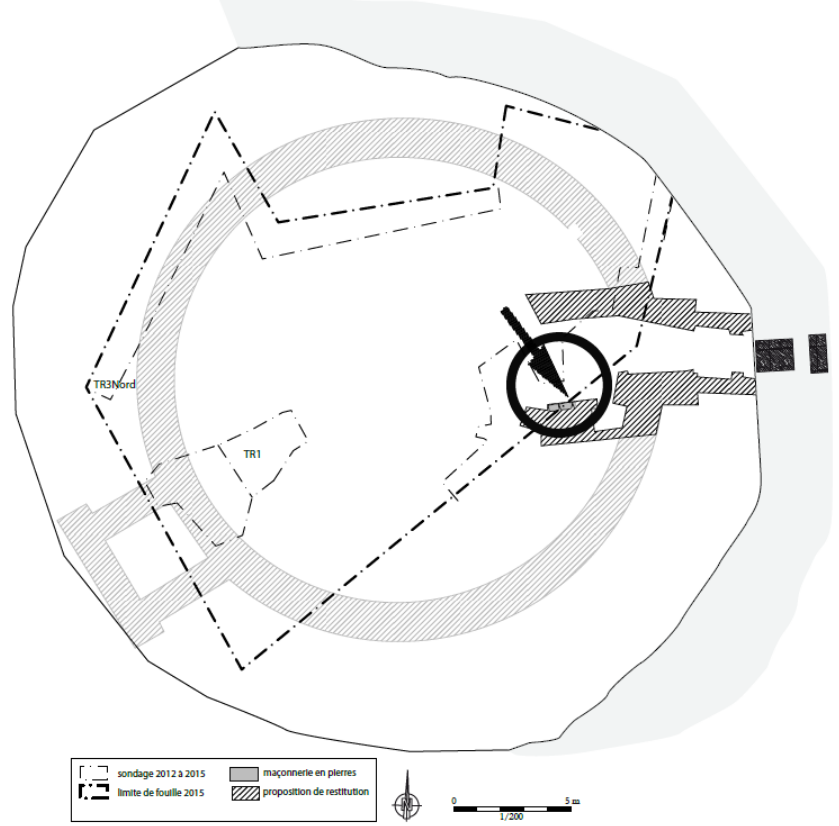

Mur en grand appareil, hypothèse de restitution $\odot$ F. Bernard et N. Béague, Inrap

Une cour intérieure circulaire d'un diamètre interne de 11 mètres délimitée par une maçonnerie en pierres couronne ensuite la motte. La fondation de mur mise au jour dans le tout premier sondage (TR1) prouve qu'un bâtiment prenait appui sur la maçonnerie de la cour intérieure pour se développer en direction de la porte d'entrée occidentale du château actuel. Donc la cour intérieure était vraisemblablement bordée de bâtiments résidentiels répartis entre la cour intérieure et une enceinte circulaire plus large (non reconnue, car hors emprise). Nous pouvons comparer ce type de résidence offrant une distribution des pièces du château tout autour de la cour centrale 
avec Restormel Castle en Cornouailles (Angleterre), probablement construite vers 1100. Dans les terres d'obédience Plantagenêt, des châteaux de type shell-keep bien connus comme Gisors, Guingamp, Clisson, Fougères et Carentan (MESQUI, 1993) se retrouvent également dans le reste du territoire français continental, mais Morlanne serait le premier château de type anglo-normand à avoir été identifié en Béarn (GARDELLES, 1972). Un double tournois de Philippe IV le Bel frappé entre 1285 et 1314, issu des terres rapportées dans la fondation du mur de la cour intérieure, nous en donne le terminus post quem.

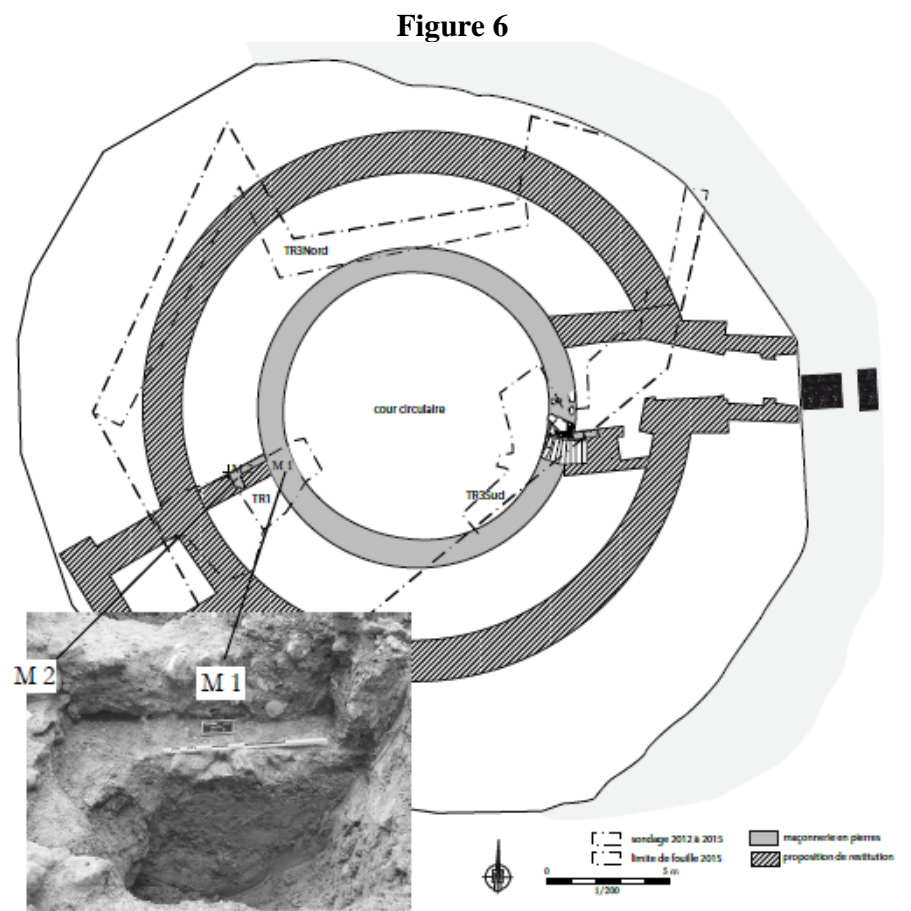

Maçonnerie de la cour intérieure hypothèse de restitution (C) F. Bernard et N. Béague, Inrap 


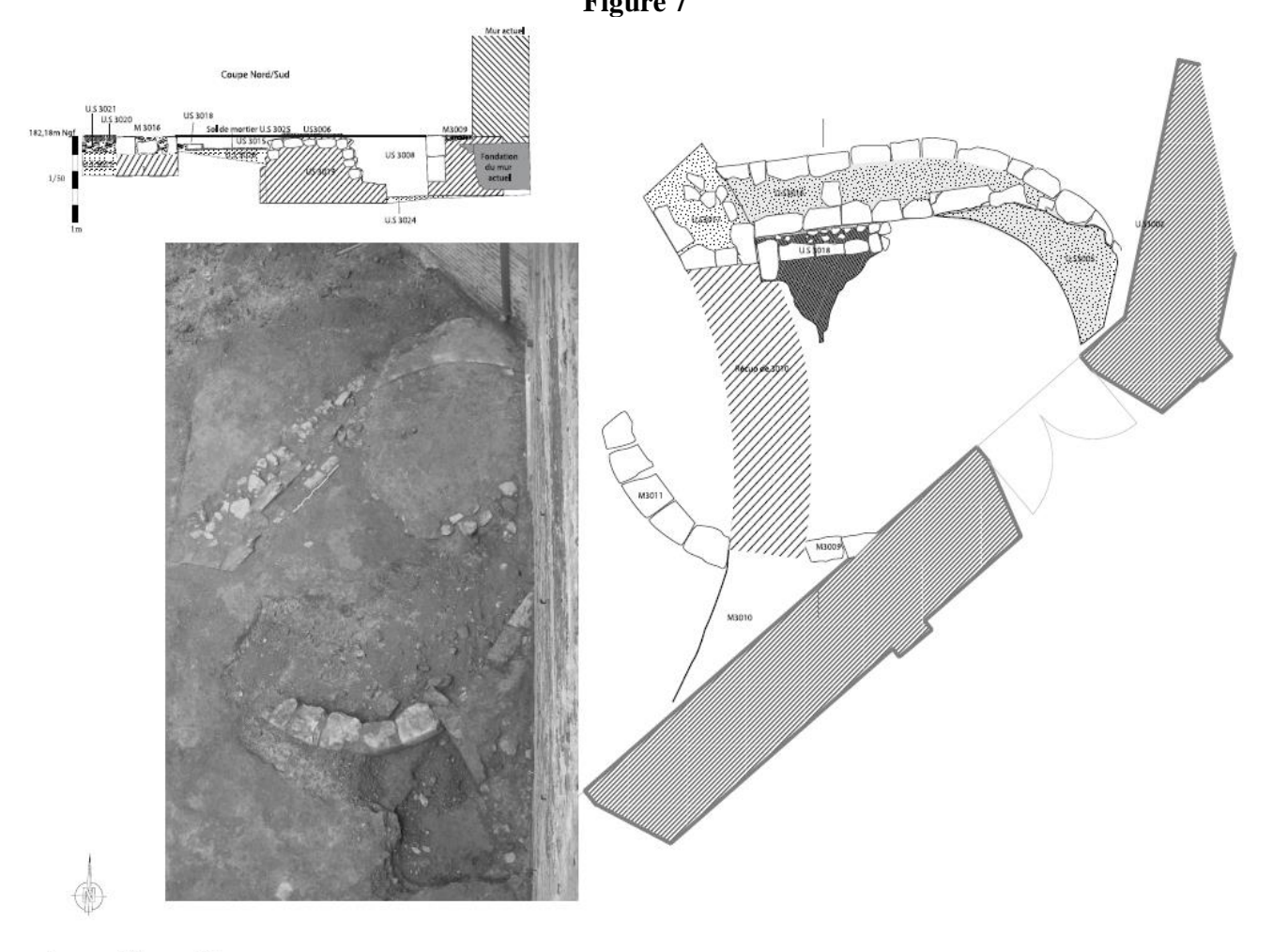

Figure 7

.

Maçonnerie en fer à cheval @ F. Bernard et N. Béague, Inrap

História: Questões \& Debates, Curitiba, volume 66, n.2, p. 45-62, jul./dez. 2018 
Figure 8

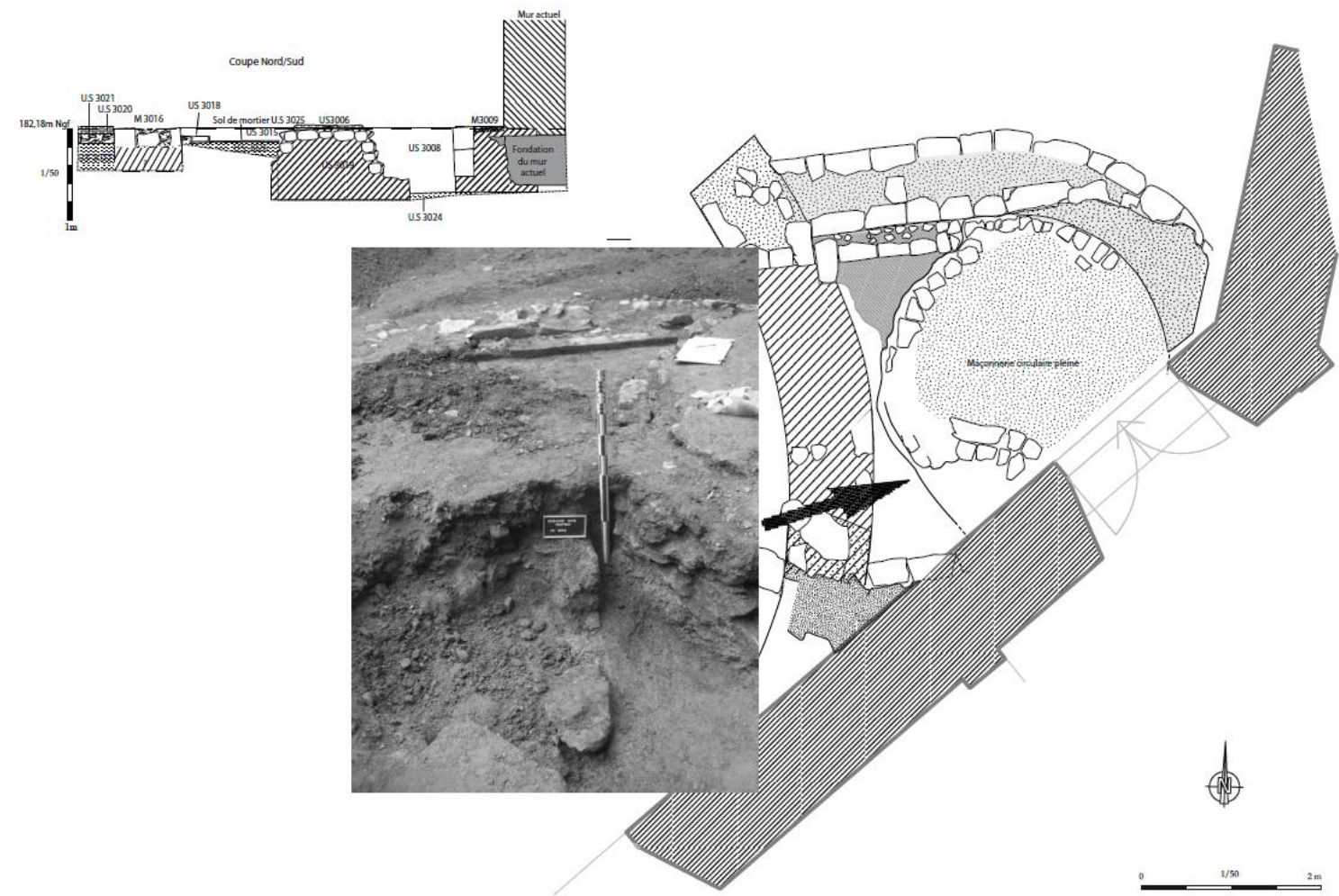

Maçonnerie de l'escalier circulaire (C) F. Bernard et N. Béague, Inrap

História: Questões \& Debates, Curitiba, volume 66, n.2, p. 45-62, jul./dez. 2018 
Une portion de la maçonnerie de cette cour circulaire est détruite au cours du XIIIe ou au début du XIVe siècle pour aménager une entrée en forme de fer à cheval (figure 7). Un escalier en vis desservait l'étage d'un bâtiment que l'on ne peut pas restituer, puisqu' il est situé à l'emplacement du château actuel (figure 8). La maçonnerie en fer à cheval est à son tour réaménagée au plus tard au milieu du XIVe siècle en tour-escalier qui devait desservir une pièce qui ne nous est pas non plus connue. Vers 1373-1374, les matériaux du château primitif en pierres sont récupérés jusqu'aux fondations pour permettre la construction du château en briques dit "fébusien" sur les ruines des états précédents. L'étude du bâti faite sur les murs intérieurs et extérieurs du château montre bien une réutilisation systématique des matériaux récupérés sur place.

\section{Figure 9}

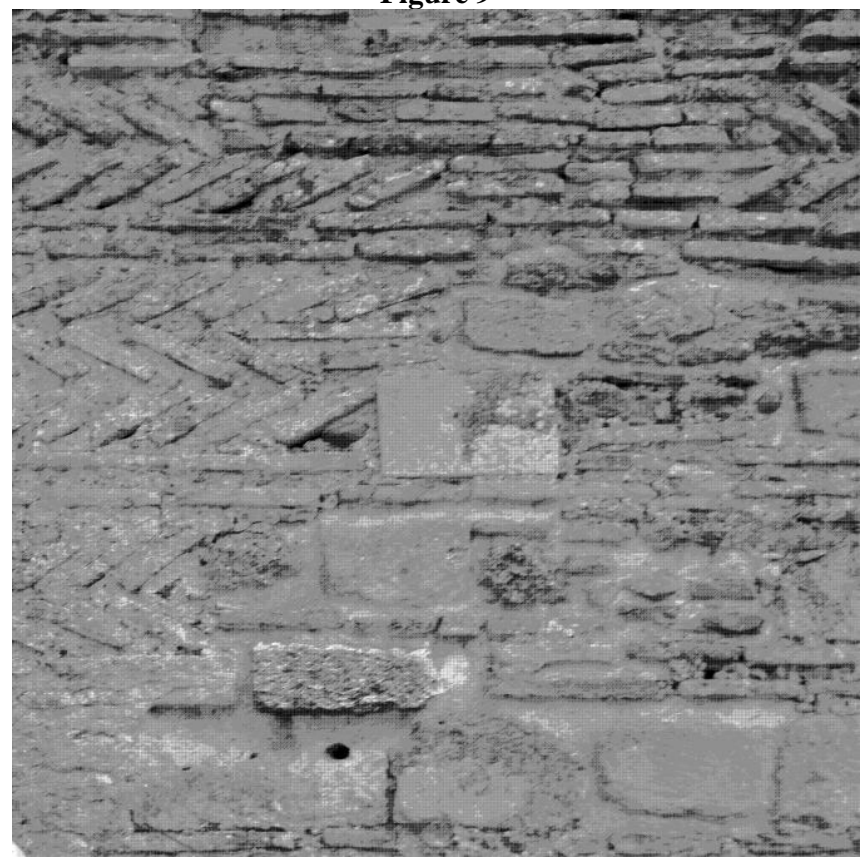

Réutilisation de matériaux plus anciens dans le château en briques (C) N. Béague, Inrap 


\section{Une légende historique démentie par l'archéologie?}

Sur quoi repose l'hypothèse de Ritter et Tucco-Chala selon laquelle Gaston Fébus aurait construit à partir des années 1370 en une dizaine d'années des châteaux d'un caractère stéréotypé, original et distinctif directement associé au nom du comte, cette œuvre de construction marquant l'apogée de l'indépendance du Béarn?

L'opération d'archéologie du bâti que nous avons réalisée parallèlement à la fouille archéologique permet de restituer l'état du château tel qu'il fut conçu à l'époque de Gaston Fébus, en faisant abstraction des multiples reconstructions qui se sont succédé pendant 600 ans. Le bâtiment d'origine donne l'image d'un château qui n'a jamais eu de vocation défensive : aucune meurtrière n'est présente en dehors de celle du donjon, les salles destinées à la réception et les chambres réservées au seigneur et à ses invités sont équipées de fenêtres à coussièges, de cheminées, de lavabos, d'éviers et de latrines. Morlanne a été construit pour le demi-frère de Gaston Fébus et non par lui dans un but de résidence. Il n'a jamais été un lieu de garnison, car il n'a jamais eu à se défendre contre l'ennemi, pas plus que le château de Montaner. Situé au centre géographique du territoire de Gaston Fébus, le château de Montaner, construit de façon certaine par Fébus, n'est pas sur une frontière à protéger! Plus qu'un élément de défense, il s'agit pour Fébus d'un élément de dissuasion, d'un symbole de domination sur son territoire et face à ses ennemis. Il n'y a d'ailleurs jamais vécu.

L'archéologie permet aussi de compléter les manques des textes à la fois pour les périodes antérieures à Gaston Fébus et contemporaines de ce grand seigneur. La fouille archéologique prouve qu'à Morlanne, un château de type anglo-normand très important par la taille et la qualité de son architecture a été habité, entretenu et agrandi avec un luxe certain pendant au moins un siècle et demi bien avant Gaston Fébus. Rien n'était connu, si ce n'est qu'un seigneur qui s'appelle Guilhem Arnaut de Morlanne est témoin du testament du vicomte de Béarn Gaston VII en 1290, puis il sert d'intermédiaire en 1319 et en 1326 entre le roi Édouard II d'Angleterre et le comte de Foix (MARCA, 1912, p. 459). Mais rien n'est précisé concernant la résidence en Béarn de ce seigneur qui a 
une position importante. En 1374, il est fait mention de Jeanne de Morlanne et de son époux Arnaud Guilhem, qui est le demi-frère de Gaston Fébus, mais là encore nous n'avons aucune indication sur leur lieu de résidence. Il est impossible de savoir d'après les textes si le château en pierres est déjà détruit ou si c'est Gaston Fébus qui ordonne de le faire raser. La fouille de la cour intérieure du château de Morlanne a permis de mettre en évidence l'extrême récupération des matériaux de construction. Les relevés du bâti montrent aussi un réemploi fréquent de matériaux datant parfois du XIIe siècle, prélevés sur place, ce qui tend à prouver que le château a été consciencieusement démonté. Il est fréquent de voir dans les textes contemporains de Gaston Fébus que des économies de moyens et de matériaux sont faites en récupérant les pierres du château précédent. Sur les chantiers des résidences vicomtales de Pau ou de Montaner, par exemple, les maçons recrutés par Gaston Fébus doivent abattre et charrier les matériaux contenus dans la tour de Morlaàs, pour les employer à la nouvelle construction. Les textes contemporains de Gaston Fébus nous montrent aussi un seigneur qui met en défense ses domaines : il oblige les habitants des villes et villages à assurer l'entretien des murs d'enceinte, il fait réparer et entretenir sa dizaine de châteaux (GALES, 2002).

Parmi les châteaux que Gaston Fébus possède en propre, beaucoup sont des réappropriations de sites acquis ou conquis par ses ancêtres : Bellocq, Labastide-Villefranche, Sauveterre-de-Béarn, le château Moncade d'Orthez, Morlaàs, Morlanne, Pau, et Montaner. Les textes prouvent que le château de Bellocq existait bien avant 1288, puisque le roi Édouard 1er d'Angleterre y est passé, mais Gaston Fébus lui a apporté des défenses supplémentaires pour abriter une garnison relativement importante et en faire la plus grande place fortifiée de la vicomté de Béarn. Ce château a été fouillé clandestinement dans les années 70, mais le rapport de fouilles ne peut rien apporter sur l'état antérieur à Gaston Fébus. Le château et le bourg de Sauveterre-de-Béarn ont été fortifiés un siècle avant Fébus par Gaston VII Moncade (1229-1290) et Fébus a ici aussi repris et complété l'ensemble fortifié du XIIIe siècle.

Le château de Mauvezin, d'architecture militaire, doit son essor à Gaston Fébus, qui s'efforça de récupérer ce château et la Bigorre dont sa famille avait été injustement spoliée en 1292. Il obtint 
Mauvezin en 1379 et le transforma en forteresse inexpugnable en faisant construire un énorme donjon carré de 37 mètres de haut sur une enceinte rectangulaire pourvue de contreforts très saillants aux angles. Il n'y a jamais eu et il n'y aura jamais de véritables fouilles archéologiques dans ce château détenu par l'association Escòla Gaston Fébus qui a en charge la restauration et la valorisation de ce monument inscrit à l'Inventaire Supplémentaire des Monuments Historiques. La présence d'un château à Navailles est mentionnée pour la première fois en 1046. Il s'agit alors d'un château " à motte " et siège de la seigneurie de Navailles, dont les seigneurs se déclarent vassaux du roi d'Angleterre Édouard Ier Plantagenêt et entretiennent des relations conflictuelles avec les vicomtes de Béarn. Les seigneurs de Navailles auraient, par goût ou poussés par Gaston Fébus, fait rebâtir leur château selon le modèle du château fébusien.

$\mathrm{Au}$ cours des années 1930, Raymond Ritter s'est souvent rendu à Navailles et il s'en est inspiré pour la restauration du château de Morlanne. Étant donné qu'il appartient à un propriétaire privé, il sera très difficile d'y faire de véritables fouilles archéologiques. Orthez, fut capitale de la vicomté de Béarn de 1242 à 1466 et a construit son identité autour de Gaston Fébus, reprenant sa devise, Toca-i si gausas (Touche si tu l'oses). Les récentes opérations archéologiques préventives que nous y avons réalisées montrent qu'un urbanisme dynamique a été mis en place plus d'un siècle avant lui. Enfin, en ce qui concerne le château de Montaner, celui-ci existait également au XIIe, car la reine d'Angleterre y a séjourné à plusieurs reprises. Gaston Fébus l'a entièrement reconstruit, sans doute comme Morlanne. Les fouilles clandestines des années 70 ont permis d'entrevoir un état ancien, mais ces travaux n'ont pas fait l'objet d'une publication. Ce château est essentiel pour la compréhension du supposé dispositif défensif de Gaston Fébus. Les textes faisant souvent défaut, seule la fouille archéologique permettrait peut-être de dire si ces châteaux étaient déjà en ruine ou ont été volontairement rasés à l'époque de Gaston Fébus. Pour savoir si leur reconstruction procède d'une réelle volonté politique et stratégique de Gaston Fébus nous allons devoir, quand cela sera possible, orienter notre travail de recherche vers une étude archéologique exhaustive.

Le château que nous avons mis au jour à Morlanne est donc contemporain de tous ces châteaux construits dès le XIIe siècle par 
des seigneurs partagés entre l'Angleterre et la France. Les textes montrent que ce sont de puissants seigneurs, engagés dans un système d'alliances et d'obligations vis-à-vis des royaumes voisins : France capétienne, Gascogne anglaise et royaume d'Aragon. Influencés par l'Angleterre, ils ont souhaité construire leur château à la mode anglaise. Fébus a-t-il justement fait démolir ces châteaux parce qu'ils étaient trop « anglais »? En prêtant à Gaston Fébus cette volonté de (re) - construire à partir des années 1370 ses châteaux suivant un caractère stéréotypé, R. Ritter et P. Tucoo-Chala ont aussi construit sa légende. C'est cette image de champion de l'indépendance du Béarn que ces historiens ont toujours voulu mettre en avant jusqu'à présent, parfois au mépris des preuves archéologiques démentant cette image.

Faut-il déconstruire la légende de Gaston Fébus ? Est-ce le rôle des archéologues de mettre en évidence une facette méconnue de l'histoire du Béarn, celle de seigneurs partagés entre l'Angleterre et la France ? L'archéologie permet de rétablir la vérité et de proposer une autre approche de la période de la guerre de Cent Ans, où tout n'était pas noir ou blanc. Il est nécessaire de nuancer nos propos vis-à-vis du public qui visite les châteaux du Béarn puisqu'il s'agit aussi bien d'enfants, de scolaires, que d'étrangers qui ne connaissent pas forcément les différents aspects de cette période de troubles, d'alliances et de traîtrises. Le public ne doit plus être considéré comme le réceptacle d'un discours péremptoire et univoque, mais doit pouvoir avoir accès aux faits établis par la recherche archéologique. Les habitants de Montaner souhaitent à l'avenir se réapproprier le site du château pour qu'à la place d'un musée à la gloire d'un seul homme ils aient un lieu de mémoire pour tous. Au château de Morlanne, le public pourra désormais être informé des dernières propositions d'interprétation par des panneaux d'information crées en collaboration avec l'Institut national de recherches archéologiques préventives (Inrap). 


\section{Conclusions}

L'archéologie préventive, qui a fait un énorme bond en avant ces 20 dernières années permet de réécrire l'Histoire (ou les histoires). Les idées évoluent à la faveur des dernières découvertes archéologiques, le public envisage plus l'archéologie comme une enquête scientifique d'experts du passé que comme une contribution à l'histoire : il attend des preuves scientifiques sur des faits tangibles. Il faut savoir s'affranchir de la personnalité écrasante de Gaston Fébus pour s'intéresser à la fois au Béarn d'avant la guerre de Cent Ans et aux anonymes qui ont fait ce que le Béarn est devenu. Maintenant que l'État est bien engagé dans la préservation du patrimoine historique, il se pourrait que les habitants commencent à être plus critiques vis-àvis des travaux effectués jusqu'à présent. Toujours réfractaires à la centralisation étatique, les Béarnais commencent à se réapproprier leurs villes, leurs châteaux et leur langue béarnaise.

\section{Bibliographie}

BÉAGUE, N. Morlanne (64), Un château en pierres avant le XIVe siècle : rapport de fouille Inrap. Bègles: CRA, 2016.

BÉAGUE, N. Morlanne, un château en pierres avant le XIVe siècle. In: A.-M. COCULA \& M. COMBET (eds.). Jeunesses et châteaux 2017, Actes des Rencontres d'Archéologie et d'Histoire en Périgord les 23, 24 et 25 septembre 2016. Bordeaux: Editions Ausonius, 2016, p. 203-215.

CHADEFAUD, M. Aux origines du tourisme dans les Pays de l'Adour. Mémoire de thèse de l'Université de Pau. Pau, France: Université de Pau et des Pays de l'Adour, 1988.

GALES, F. Les résidences de Gaston Fébus en Béarn, Résidences aristocratiques, résidences du pouvoir entre Loire et Pyrénées Xe- 
XVe siècles. Actes du Colloque de Pau 3-5 Octobre 2002. Archéologie du Midi Médiéval, Supplément 4, 2002.

GARDELLES, J. Les châteaux du Moyen Âge dans la France du SudOuest. La Gascogne anglaise de 1216 à 1327. Arts et métiers graphiques, IN-4, Collection Bibliothèque de la société française d'archéologie, n 3, 1972, 288 p.

MARCA, P. de. : Histoire de Béarn. In : V. Dubarat (ed.). Pau : Ribaut, [1640] 1912 .

MESQUI, Jean. Châteaux et enceintes de la France médiévale. De la défense à la résidence, 2 volumes. Paris: Picard, 1993.

Ritter, Raymond. Châteaux, donjons et places fortes. L'architecture militaire française. Paris: Larousse, 1953.

TUCOO-CHALA, P. Gaston Fébus : prince des Pyrénées : 1331 1391. Biarritz : J \& D Editions, 1993.

RECEBIDO EM: 01/06/2018 APROVADO EM: 12/07/2018 